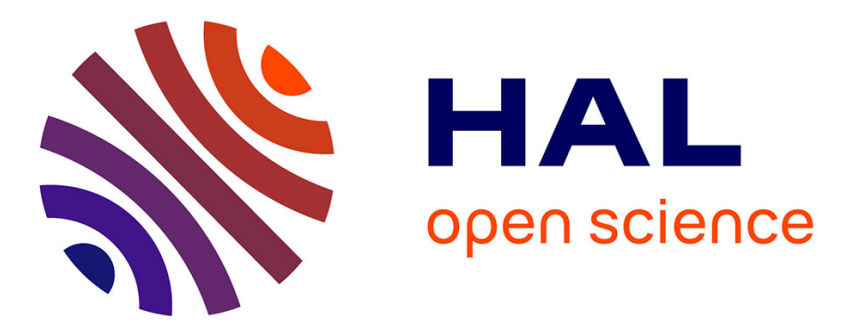

\title{
Distributed estimation based on multi-hop subspace decomposition
}

Alvaro Rodrigues del Nozal, Pablo Millan Gata, Luis Orihuela, Alexandre Seuret, Luca Zaccarian

\section{- To cite this version:}

Alvaro Rodrigues del Nozal, Pablo Millan Gata, Luis Orihuela, Alexandre Seuret, Luca Zaccarian. Distributed estimation based on multi-hop subspace decomposition. Automatica, 2019, 99, pp.213220. 10.1016/j.automatica.2018.10.034 . hal-01920417

\section{HAL Id: hal-01920417 https://hal.laas.fr/hal-01920417}

Submitted on 13 Nov 2018

HAL is a multi-disciplinary open access archive for the deposit and dissemination of scientific research documents, whether they are published or not. The documents may come from teaching and research institutions in France or abroad, or from public or private research centers.
L'archive ouverte pluridisciplinaire HAL, est destinée au dépôt et à la diffusion de documents scientifiques de niveau recherche, publiés ou non, émanant des établissements d'enseignement et de recherche français ou étrangers, des laboratoires publics ou privés. 


\title{
Distributed estimation based on multi-hop subspace decomposition
}

\author{
A.R. del Nozal ${ }^{\text {a }}$, P. Millán ${ }^{\text {a }}$, L. Orihuela ${ }^{\text {a }}$, A. Seuret ${ }^{\text {b }}$, L. Zaccarian ${ }^{\text {b,c }}$ \\ ${ }^{a}$ Departamento de Ingeniería, Universidad Loyola Andalucía, Sevilla, Spain \\ ${ }^{\mathrm{b}} L A A S-C N R S$, Université de Toulouse, CNRS, Toulouse, France \\ ${ }^{\mathrm{c}}$ Department of Industrial Engineering, University of Trento, Trento, Italy
}

\begin{abstract}
This paper deals with the problem of distributedly estimating the state of an LTI plant through an interconnected network of agents. The proposed approach results in an observer structure that incorporates consensus among the agents and that can be distributedly designed, achieving a robust solution with a good estimation performance. The developed solution is based on an iterative decomposition of the plant in the local observable staircase forms. The proposed observer has several positive features compared to recent results in the literature, which include milder assumptions on the network connectivity and the ability to set the convergence rate.
\end{abstract}

Key words: Distributed estimation, Multi-agent systems, Time-invariant systems, Linear systems.

\section{Introduction}

Nowadays, the increasing number of embedded sensors dispersedly integrated in complex plants is fostering the application of distributed estimation and control schemes. Although centralized strategies may benefit from information collected throughout a network, distributed strategies offer interesting advantages such as scalability, flexibility, fault tolerance, and robustness [21]. Despite the aforementioned advantages, the complexity associated with distributed observation problems makes them harder to solve, since additional difficulties take place.

Under the assumptions of stochastic, independent, and identically distributed noises and disturbances, the most well-known approach to this problem is the distributed Kalman filter (DKF) first presented in [17], sometimes combined with consensus algorithms [2], or extended to nonlinear systems [1]. Consensus techniques are also

\footnotetext{
ऋ Research partially supported by grant TEC2016-80242-P founded by AEI/FEDER

Email addresses: arodriguez@uloyola.es (A.R. del Nozal), pmillan@uloyola.es (P. Millán), dorihuela@uloyola.es (L. Orihuela), aseuret@laas.fr (A. Seuret), zaccarian@laas.fr (L. Zaccarian).
}

used in [10], where the estimates of the neighboring sensors are weighted by the inverse of their covariance error matrices. Diffusion strategies for distributed Kalman filtering are studied in [3]. A different approach to the same problem can be found in [22], where the convergence time of the DKF to the solution provided by the Centralized Kalman Filter is studied. [4] presents a subspacedecomposition-based observer, whose aim is to minimize the exchange of information during the estimation phase.

Furthermore, there are a number of distributed estimation techniques proposed and analysed for noiseless models. In [19], [20], and [?], the distributed estimation problem is tackled as the problem of designing a decentralized stabilizing controller for an LTI system. Differently from the approach presented in this paper, these works rely on state augmentations. Another interesting approach can be found in [11], where the authors rely on an orthonormal coordinate transformation matrix in order to tackle the design of the distributed observer. However, the distributed design of the observer needs global information about the communication graph, and the proposed observer lacks of a tuning method to adjust the error convergence speed. In [7], a distributed observer is designed resorting to linear matrix inequalities, and asymptotic decay of the estimation with a prescribed decay rate is guaranteed. Finally, in [15], the authors design a distributed observer assigning observable 
subspaces to each agent, based on its measurements of the plant and on a random distribution of identification numbers. However, the design method does not allow to tune the convergence rate and the distributed design requires a huge amount of information exchange.

This paper proposes a novel approach to this problem based on a decomposition of the state-space in orthogonal subspaces that capture the locally unobservable modes of each agent involved in the network. This work extends our preliminary result in [12], where the number of agents was limited to two. The observers use local information measured from the plant to correct the locally observable subspace, whereas the locally unobservable subspace is divided according to the innovations introduced by neighboring agents, which are incorporated in the observers' dynamics through a consensus term. The measurements and network connectivity requirements are encapsulated in a single assumption, which makes it possible to relax the common assumptions of strongly connected graphs, included for instance in [7], or strongly connected graph components, in [15] or [?]. A different approach can be found in [5] and [6] where the authors make use of structural systems theory to establish conditions for distributed observability. Since the conditions presented in [5] depend on the particular estimator structure, they fail to provide necessary and sufficient conditions, which are instead established in the present paper by using just the system dynamics, the measurements and the communication topology.

Unlike the state augmentation approach in [19], [20] and $[?]$, the proposed method does not require state augmentation or the resolution of linear matrix inequalities, which reduces the computational costs. More importantly, and differently from [11], [13] and [14], the design of the observers are carried out in a distributed way, which is crucial for large scale networks or time-varying topologies. Differently from [11] or [15], the presented design method provides flexibility to adjust the convergence rate of the estimation dynamics. Other positive features are that the distributed design can be carried out with a reasonably low information exchange, and that, once the estimators have been designed, the communication requirements are even lower, as the agents only need to communicate certain subspaces. Finally, the paper demonstrates that the proposed design is always feasible under necessary assumptions.

The paper is organized as follows. Section 2 introduces models for the plant, the network of agents, and the information structure, together with some definitions needed to understand the rest of the paper. Section 3 presents the proposed observation structure together with the standing assumptions and the design goal. In Section 4, the stability analysis of the error dynamics is presented. Section 5 extends the algorithm to the continuous-time case. Section 6 presents an algorithm to build the estimation structure of the agents. Section
7 shows the observer performance. Finally, conclusions are drawn in Section 8.

Notation. A graph is a pair $\mathcal{G}=(\mathcal{V}, \mathcal{E})$ comprising a set $\mathcal{V}=\{1,2, \ldots, p\}$ of vertices or agents, and a set $\mathcal{E} \subset \mathcal{V} \times \mathcal{V}$ of edges or links. A directed graph is a graph in which edges have orientations, so that if $(j, i) \in \mathcal{E}$, then agent $i$ obtains information from agent $j$. A directed path from node $i_{1}$ to node $i_{k}$ is a sequence of edges such as $\left(i_{1}, i_{2}\right),\left(i_{2}, i_{3}\right), \ldots,\left(i_{k-1}, i_{k}\right)$ in a directed graph. The neighborhood of $i, \mathcal{N}_{i} \triangleq\{j:(j, i) \in \mathcal{E}\}$, is defined as the set of nodes with edges incoming to node $i$. Given $\rho \in \mathbb{Z}>0$, the $\rho$-hop reachable set of $i, \mathcal{N}_{i, \rho}$, is defined as the set of nodes with a direct path to $i$ involving $\rho$ edges. Note that the 1-hop reachable set of $i$ corresponds to the neighborhood of $i$. The operator $\operatorname{col}(\cdot, \cdot)$ stacks subsequent matrices into a column vector, e.g. for $A \in \mathbb{R}^{m_{1} \times n}$ and $B \in \mathbb{R}^{m_{2} \times n}, \operatorname{col}(A, B)=\left[A^{\top} B^{\top}\right]^{\top} \in$ $\mathbb{R}^{\left(m_{1}+m_{2}\right) \times n}$. Pair $(C, A)$ is $\alpha$-detectable if $\left(C, \alpha^{-1} A\right)$ is detectable, namely if the unobservable modes have exponential convergence at least equal to $\alpha . \operatorname{Im}(A)$ denotes the image of matrix $A$, i.e., the subspace generated by the columns of matrix $A$. A collection of subspaces $\left\{\operatorname{Im}\left(W_{1}\right), \ldots, \operatorname{Im}\left(W_{n}\right)\right\}$ is independent if no nonzero column of $W_{i}$ is a linear combination of some columns of the rest of matrices $W_{j}$, for all $j \neq i$. The sum of two subspaces $\operatorname{Im}\left(W_{1}\right)$ and $\operatorname{Im}\left(W_{2}\right)$ is denoted by $\operatorname{Im}\left(W_{1}\right)+$ $\operatorname{Im}\left(W_{2}\right)=\left\{w_{1}+w_{2} \mid w_{1} \in \operatorname{Im}\left(W_{1}\right), w_{2} \in \operatorname{Im}\left(W_{2}\right)\right\}$. The sum of $\operatorname{Im}\left(W_{1}\right)$ and $\operatorname{Im}\left(W_{2}\right)$ is direct if $\operatorname{Im}\left(W_{1}\right) \cap$ $\operatorname{Im}\left(W_{2}\right)=\{0\}$ and is denoted by $\operatorname{Im}\left(W_{1}\right) \oplus \operatorname{Im}\left(W_{2}\right)$.

\section{Preliminaries and multi-hop decomposition}

Consider a set of agents $\mathcal{V}=\{1,2, \ldots, p\}$ connected according to a given graph $\mathcal{G}=(\mathcal{V}, \mathcal{E})$, and intended to distributedly estimate the state of the following discretetime LTI system:

$$
\begin{aligned}
& x^{+}=A x \\
& y_{i}=C_{i} x \quad \forall i \in \mathcal{V}
\end{aligned}
$$

where $x$ is the state vector, $A$ is the system matrix, $y_{i} \in \mathbb{R}^{m_{i}}$ is the output locally measured by each agent $i$ and $C_{i} \in \mathbb{R}^{m_{i} \times n}$ is the output matrix of agent $i$.

The observation structure proposed in the next section relies on system transformations to the observability staircase form (see for instance Theorem 16.2 in [8]). Prior to introducing this structure, the following definitions are needed.

Definition 1 The $\rho$-hop output matrix of agent $i, C_{i, \rho}$, is a matrix that stacks the $(\rho-1)$-hop output matrix of agent $i$ and the $(\rho-1)$-hop output matrices of its neighborhood, $\mathcal{N}_{i}$. That is: 


$$
C_{i, \rho}:=\left[\begin{array}{c}
C_{i, \rho-1} \\
\operatorname{col}\left(C_{j, \rho-1}\right)_{j \in \mathcal{N}_{i}}
\end{array}\right], \quad \forall \rho \geq 1,
$$

where $C_{i, 0}:=C_{i}$.

Intuitively speaking, the $\rho$-hop output matrix of agent $i, C_{i, \rho}$, is composed by its output matrix $C_{i}$ and the output matrices of all the agents $j$ with a direct path to $i$ involving $\rho$ or less edges.

There always exists a coordinate transformation matrix $\left[\bar{V}_{i, \rho} V_{i, \rho}\right] \in \mathbb{R}^{n \times n}$ according to pair $\left(C_{i, \rho}, A\right)$ such that the change of variable $\xi_{i, \rho} \triangleq\left[\bar{V}_{i, \rho} V_{i, \rho}\right]^{\top} x \in \mathbb{R}^{n}$ transforms the original state-space representation into the observability staircase form. Note that $\bar{V}_{i, \rho} \in \mathbb{R}^{n \times n_{i, \rho}^{\bar{o}}}$ is composed by $n_{i, \rho}^{\bar{o}}$ column vectors in $\mathbb{R}^{n}$ that form an orthogonal basis of the unobservable subspace of pair $\left(C_{i, \rho}, A\right)$. Correspondingly, $V_{i, \rho} \in \mathbb{R}^{n \times n_{i, \rho}^{o}}$ is an orthogonal basis of its orthogonal complement.

Definition 2 The $\rho$-hop unobservable subspace from agent $i$, denoted $\overline{\mathcal{O}}_{i, \rho}$, is composed of all system modes that cannot be observed from the output locally measured by agent $i$ and those measured by all the agents belonging to the s-hop reachable set of $i, \forall s \in\{0, \ldots, \rho\}$. Equivalently, the $\rho$-hop unobservable subspace from agent $i$ is the unobservable subspace related to pair $\left(C_{i, \rho}, A\right)$ using the above coordinate transformation:

$$
\overline{\mathcal{O}}_{i, \rho}:=\operatorname{Im}\left(\bar{V}_{i, \rho}\right) .
$$

The orthogonal complement of $\overline{\mathcal{O}}_{i, \rho}$, with some abuse of notation, is denoted $\rho$-hop observable subspace from agent $i, \mathcal{O}_{i, \rho}:=\operatorname{Im}\left(V_{i, \rho}\right)$. We denote $n_{i, \rho}^{o}=\operatorname{dim}\left(\mathcal{O}_{i, \rho}\right)$.

According to Definition 2, it is clear that:

$$
\mathcal{O}_{i, \rho-1} \subseteq \mathcal{O}_{i, \rho}, \quad \forall i \in \mathcal{V}, \quad \rho \geq 0 .
$$

where we consider $\mathcal{O}_{i,-1}=\emptyset$. Then, the vectors of the "innovation" basis that generates $\mathcal{O}_{i, \rho} \cap\left(\mathcal{O}_{i, \rho-1}\right)^{\perp}$ can be stacked into a matrix $W_{i, \rho} \in \mathbb{R}^{n \times n_{i, \rho}}$, where $n_{i, \rho}=$ $n_{i, \rho}^{o}-n_{i, \rho-1}^{o}$, in such a way that:

$$
\operatorname{Im}\left(W_{i, \rho}\right):=\mathcal{O}_{i, \rho} \cap\left(\mathcal{O}_{i, \rho-1}\right)^{\perp}, \quad \rho \geq 0,
$$

Let us, to be selected later, define $\ell_{i} \in \mathbb{Z}_{>0}$ as an arbitrary number of hops. From these definitions it is clear that for all $\rho \in\left\{0, \ldots, \ell_{i}\right\}$ and all $i \in \mathcal{V}$, it holds that

$$
\begin{aligned}
& \operatorname{Im}\left(V_{i, \rho}\right)=\operatorname{Im}\left(\left[W_{i, \rho} V_{i, \rho-1}\right]\right), \\
& \operatorname{Im}\left(\bar{V}_{i, \rho-1}\right)=\operatorname{Im}\left(\left[\begin{array}{ll}
W_{i, \rho} & \bar{V}_{i, \rho}
\end{array}\right]\right),
\end{aligned}
$$

with $\bar{V}_{i,-1}:=I_{n}$.
It is worth pointing out that $\operatorname{Im}\left(W_{i, \rho}\right)$ corresponds to the innovation introduced by the $\rho$-hop reachable set $\mathcal{N}_{i, \rho}$ of agent $i$, that is, the observable modes for agent $i$ at hop $\rho$ that are not observable at hop $\rho-1$. Accordingly, the transformation matrix $T_{i}$, defined as $T_{i}=\left[\begin{array}{l}\bar{V}_{i, \ell_{i}} V_{i, \ell_{i}}\end{array}\right]$, can be divided using the innovations at each hop:

$$
T_{i}:=[\underbrace{\bar{V}_{i, \ell_{i}} W_{i, \ell_{i}} \cdots W_{i, \rho+1}}_{\bar{V}_{i, \rho}} \underbrace{W_{i, \rho} \cdots W_{i, 0}}_{V_{i, \rho}}] \in \mathbb{R}^{n \times n},
$$

for all $\rho \in\left\{0, \ldots, \ell_{i}\right\}$, where it is easy to identify the observable and unobservable subspaces of the system by agent $i$ at hop $\rho$.

The following lemma introduces some important properties that are central for the subsequent derivations.

Lemma 3 For any agent $i \in \mathcal{V}$, the next properties hold, $\forall \rho, \rho^{\prime} \in\left\{1, \ldots, \ell_{i}\right\}$ such that $\rho \neq \rho^{\prime}$ :

(i) $W_{i, \rho}^{\top} W_{i, \rho^{\prime}}=0$,

(ii) $\operatorname{Im}\left(W_{j, \rho-1}\right) \subseteq \operatorname{Im}\left(V_{i, \rho}\right), \forall j \in \mathcal{N}_{i}$,

(iii) $\operatorname{Im}\left(W_{i, \rho}\right) \subseteq \underset{j \in \mathcal{N}_{i}}{\operatorname{Im}}\left(W_{j, \rho-1}\right)$,

Proof of (i): Take any $\rho \neq \rho^{\prime}$ and assume without loss of generality $\rho>\rho^{\prime}$. From (4) we have $\operatorname{Im}\left(W_{i, \rho}\right) \subseteq\left(\mathcal{O}_{i, \rho-1}\right)^{\perp}$ and $\operatorname{Im}\left(W_{i, \rho-1}\right) \subseteq \mathcal{O}_{i, \rho-1}$ and then, $W_{i, \rho}^{\top} W_{i, \rho-1}=0$. Applying (3) recursively we obtain that $\operatorname{Im}\left(W_{i, \rho}\right)$ is orthogonal to $\operatorname{Im}\left(W_{i, \rho^{\prime}}\right)$ for all $\rho^{\prime}>\rho$, which proves item (i).

Proof of (ii): From Definition 2, we have that pairs $\left(C_{i, \rho}, A\right)$ and $\left(C_{j, \rho-1}, A\right)$ generate subspaces $\mathcal{O}_{i, \rho}$ and $\mathcal{O}_{j, \rho-1}$ respectively. Then, Definition 1 ensures that matrix $C_{j, \rho-1}$ is one of the stacked matrices in $C_{i, \rho}$, which clearly implies $\mathcal{O}_{j, \rho-1} \subseteq \mathcal{O}_{i, \rho}$. Finally, from (4) we have $\operatorname{Im}\left(W_{j, \rho-1}\right) \subseteq \mathcal{O}_{j, \rho-1}$ and, consequently, $\operatorname{Im}\left(W_{j, \rho-1}\right) \subseteq$ $\mathcal{O}_{i, \rho}$ establishing item (ii).

Proof of (iii): We follow the same reasoning as for (ii). From Definition 1, we know that matrix $C_{i, \rho}$ is composed by matrix $C_{i, \rho-1}$ and matrices $C_{j, \rho-1}$ for every neighbor $j$ of agent $i$. From Definition 2, it is easy to check that $\mathcal{O}_{i, \rho}=\operatorname{Im}\left(V_{i, \rho-1}\right) \underset{j \in \mathcal{N}_{i}}{\bigoplus}\left(\operatorname{Im}\left(V_{j, \rho-1}\right)\right)$. Moreover, (4) implies that $\operatorname{Im}\left(W_{i, \rho}\right) \subseteq\left(\mathcal{O}_{i, \rho-1}\right)^{\perp}$ and $\operatorname{Im}\left(W_{i, \rho}\right) \subseteq \mathcal{O}_{i, \rho}$, and, consequently, $\operatorname{Im}\left(W_{i, \rho}\right) \subseteq$ $\bigoplus_{j \in \mathcal{N}_{i}} \operatorname{Im}\left(V_{j, \rho-1}\right)$, which are the subspaces generated $\bigoplus_{j \in \mathcal{N}_{i}}$

by output matrices $C_{j, \rho-1}$. Note that matrices $C_{j, \rho-2}$ are a part of $C_{i, \rho-1}$ so that $\mathcal{O}_{j, \rho-2} \subseteq \mathcal{O}_{i, \rho-1}$. This implies, using $\operatorname{Im}\left(W_{i, \rho}\right) \subseteq\left(\mathcal{O}_{i, \rho-1}\right)^{\perp}$ again, that $\operatorname{Im}\left(W_{i, \rho}\right) \subseteq\left(\mathcal{O}_{j, \rho-2}\right)^{\perp}$ for every neighbor $j$ of $i$. Using (6), $\left(\mathcal{O}_{j, \rho-2}\right)^{\perp}=\operatorname{Im}\left(\left[W_{j, \rho-1} \bar{V}_{j, \rho-1}\right]\right)$ and 
then $\operatorname{Im}\left(W_{i, \rho}\right) \subseteq \bigoplus_{j \in \mathcal{N}_{i}} \operatorname{Im}\left(\left[W_{j, \rho-1} \bar{V}_{j, \rho-1}\right]\right)$. Since $\operatorname{Im}\left(V_{j, \rho-1}\right) \cap \operatorname{Im}\left(\bar{V}_{j \rho-1}\right)=\emptyset$ by definition, then item (iii) is proven.

\section{Observer structure and design goal}

This section presents a novel observer structure that makes use of the notions previously introduced. In particular, the proposed observer structure is as follows:

$$
\begin{aligned}
\widehat{x}_{i}^{+} & =\underbrace{A \widehat{x}_{i}}_{(a)}+\underbrace{W_{i, 0} L_{i}\left(y_{i}-\widehat{y}_{i}\right)}_{(b)}+ \\
& +\underbrace{\sum_{\rho=1}^{\ell_{i}} \sum_{j \in \mathcal{N}_{i}} W_{i, \rho} N_{i, j, \rho} W_{j, \rho-1}^{\top}\left(\widehat{x}_{j}-\widehat{x}_{i}\right)}_{(c)}
\end{aligned}
$$

where $L_{i}$ and $N_{i, j, \rho}$ are, respectively, a local observer gain and consensus gains to be designed. The observation structure proposed in (8) decomposes the observer dynamics in three different terms:

(a) The first one, $A \hat{x}_{i}$, is the classical model-based openloop prediction.

(b) The second term, containing $L_{i}\left(y_{i}-\widehat{y}_{i}\right)$, is a local Luenberger-like output injection term, intended to correct the previous prediction with the difference between the local measures and its predicted outputs $\widehat{y}_{i}:=C_{i} \hat{x}_{i}$. It is worth noting that this term is pre-multiplied by $W_{i, 0}$, which implies that the elements in the correction vector $L_{i}\left(y_{i}-\hat{y}_{i}\right)$ are actually used as weights to perform linear combinations of the column vectors forming $W_{i, 0}$. Thus, these corrections only affect the observable subspace of agent $i$. This makes full sense, as the locally available output $y_{i}$ only contains information about this subspace.

(c) This last term aims at adjusting the estimates $\hat{x}_{i}$ with the information received by the neighboring agents. Thus, the differences between the estimates of $i$ and $j$ are multiplied by matrix $W_{j, \rho-1}^{\top}$. The result is multiplied by gain matrix $N_{i, j, \rho}$ and is used as weights to perform linear combinations of $W_{i, \rho}$. It is worth mentioning that each neighbor $j$ can compute and exchange $W_{j, \rho-1} \hat{x}_{j}$, whose dimension is smaller than $\hat{x}_{j}$, reducing in this way the exchange of information through the network.

The goal of this paper is to design the gain matrices $L_{i}$ and $N_{i, j, \rho}$ to solve the following problem:

Problem 4 (Distributed $\alpha$-estimation) Given $\alpha \in(0,1)$, plant $(1)-(2)$, and the interconnection graph $\mathcal{G}=(\mathcal{V}, \mathcal{E})$, design the gains $L_{i}$ and $N_{i, j, \rho}$ in (8) such that all estimates $\hat{x}_{i}$ converge to $x$ exponentially fast with exponential rate $\alpha$.
Next, we include a definition and a necessary assumption for the solvability of Problem 4.

Definition 5 Given $\alpha \in(0,1)$, pair $(C, A)$ is $\alpha$ detectable if pair $(C, A / \alpha)$ is detectable (in the sense of [8, Def 16.1]). Moreover, system (1)-(2) is collectively $\alpha$-detectable if for each agent $i \in \mathcal{V}$, there exists a finite number of hops $\ell_{i} \in \mathbb{Z}>0$ such that pair $\left(C_{i, \ell_{i}}, A\right)$ is $\alpha$-detectable.

By definition, we see that a pair $(C, A)$ is $\alpha$-detectable if and only if the unobservable modes of the observable decomposition have convergence rate of at least $\alpha$, namely if and only if there exists an observer ensuring the exponential stabilization of the estimation error with convergence rate $\alpha$. Similarly, system (1)-(2) is collectively detectable if for each agent, the complete information provided by the network (that is, the $\rho$-hop output matrix with $\rho$ arbitrarily large) is sufficient to build such an observation law. Due to this fact, collective $\alpha$-detectability is necessary to solve Problem 4 and is assumed next.

Assumption 6 Given $\alpha \in(0,1)$, we assume that system (1)-(2) is collectively $\alpha$-detectable.

Remark 7 If the communication graph is connected and pair $\left(\left[C_{1}^{\top}, \ldots, C_{p}^{\top}\right]^{\top}, A\right)$ is $\alpha$-detectable (as, for instance, in [7] and [24]), then Assumption 6 holds true. However, Assumption 6 is in general less restrictive, as it does not enforce connectivity of the network (see for instance the example in Figure 1).

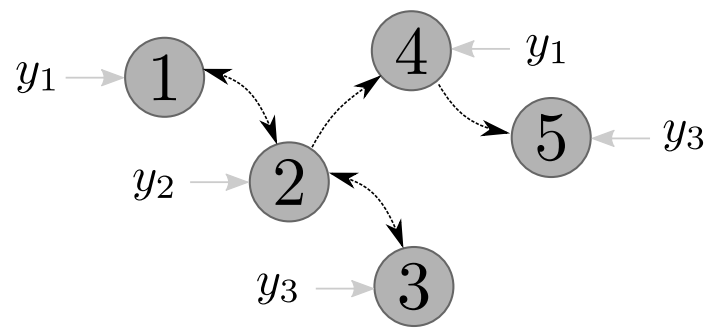

Fig. 1. Assume that pair $(\tilde{C}, A)$ with $\tilde{C}=\left[C_{1}^{\top}, C_{2}^{\top}, C_{3}^{\top}\right]^{\top}$ is $\alpha$-detectable. Although strong connectivity does not hold, Assumption 6 is still met.

\section{Design and stability of the distributed ob- server}

This section presents a design method for the distributed observers that guarantees stability with prescribed convergence rate. First, we need to introduce the following result, based on Lemma 5.49 in [18] (whose straightforward proof is omitted), which is necessary to establish the subsequent proposition.

Lemma 8 If $\operatorname{Im}\left(\bar{V}_{i, \rho}\right) \subseteq \overline{\mathcal{O}}_{i, \rho}$, then $\operatorname{Im}\left(A \bar{V}_{i, \rho}\right) \subseteq \overline{\mathcal{O}}_{i, \rho}$, i.e., the unobservable subspace $\overline{\mathcal{O}}_{i, \rho}$ is an A-invariant subspace. 
Proposition 9 For each agent $i$, the orthogonal similarity transformation given by $T_{i}$ in (7) transforms the system matrix $A$ into a block upper-triangular matrix in the form:

$$
\begin{aligned}
& T_{i}^{\top} A T_{i}= \\
& {\left[\begin{array}{ccccc}
\bar{V}_{i, \ell_{i}}^{\top} A \bar{V}_{i, \ell_{i}} & \bar{V}_{i, \ell_{i}}^{\top} A W_{i, \ell_{i}} & \cdots & \bar{V}_{i, \ell_{i}}^{\top} A W_{i, 1} & \bar{V}_{i, \ell_{i}}^{\top} A W_{i, 0} \\
0 & W_{i, \ell_{i}}^{\top} A W_{i, \ell_{i}} & \cdots & W_{i, \ell_{i}}^{\top} A W_{i, 1} & W_{i, \ell_{i}}^{\top} A W_{i, 0} \\
\vdots & \vdots & \ddots & \vdots & \vdots \\
0 & 0 & \cdots & W_{i, 1}^{\top} A W_{i, 1} & W_{i, 1}^{\top} A W_{i, 0} \\
0 & 0 & \cdots & 0 & W_{i, 0}^{\top} A W_{i, 0}
\end{array}\right] .}
\end{aligned}
$$

PROOF. The transformation matrix defined in (7) is composed of the vectors that form a basis of the innovations $W_{i, \rho}$, introduced by the neighbors of agent $i$ at each hop $\rho$, and of those that form the basis of the unobservable subspace at hop $\ell_{i}$, which, according to Assumption 6 , must be $\alpha$-detectable by the network. Note that from Lemma 3 (i), all the innovation terms are mutually orthogonal and therefore $T_{i}$ is a full rank transformation matrix.

Applying transformation $T_{i}$ to the dynamics matrix of the system focusing on the partition related to hop $\rho$ in (7), the next expression is obtained for (9):

$$
T_{i}^{\top} A T_{i}=\left[\begin{array}{ll}
\bar{V}_{i, \rho}^{\top} A \bar{V}_{i, \rho} & \bar{V}_{i, \rho}^{\top} A V_{i, \rho} \\
V_{i, \rho}^{\top} A \bar{V}_{i, \rho} & V_{i, \rho}^{\top} A V_{i, \rho}
\end{array}\right]
$$

Then, according to Lemma 8, $\operatorname{Im}\left(A \bar{V}_{i, \rho}\right) \subseteq \operatorname{Im}\left(\bar{V}_{i, \rho}\right)$ which clearly implies $V_{i, \rho}^{\top} A \bar{V}_{i, \rho}=0$, and therefore:

$$
T_{i}^{\top} A T_{i}=\left[\begin{array}{c|c}
\bar{V}_{i, \rho}^{\top} A \bar{V}_{i, \rho} & \bar{V}_{i, \rho}^{\top} A V_{i, \rho} \\
\hline 0 & V_{i, \rho}^{\top} A V_{i, \rho}
\end{array}\right]
$$

which is valid for every considered hop $\rho$. Applying this procedure recursively from $\rho=0$ to $\rho=\ell_{i}$, it is clear that the diagonal elements correspond to $W_{i, \rho}^{\top} A W_{i, \rho}$ whereas each term below the diagonal is zero, which establishes (9).

Note that the first block row of matrix (9) corresponds to those modes that are unobservable but $\alpha$-detectable.

Let us define the estimation error of any agent $i$ as:

$$
e_{i}:=x-\hat{x}_{i}, \quad i \in \mathcal{V}
$$

Similarly, it is possible to define the transformed estimation error as $\varepsilon_{i}:=T_{i}^{\top} e_{i}$. More specifically, the estimation error of agent $i \in \mathcal{V}$, at hop $\rho$, is defined as:

$$
\varepsilon_{i, \rho}:=W_{i, \rho}^{\top} e_{i}, \quad \forall \rho=0, \ldots, \ell_{i}+1,
$$

where we denote $W_{i, \ell_{i}+1}=\bar{V}_{i, \ell_{i}}$ corresponding to the collectively unobservable but detectable system modes. The following lemma will be useful later on.

Lemma 10 Under Assumption 6, the next equation holds for any $i \in \mathcal{V}$, any $j \in \mathcal{N}_{i}$, and any $\rho \in\left\{0, \ldots, \ell_{i}\right\}$

$$
\begin{aligned}
& W_{j, \rho-1}^{\top}\left(\hat{x}_{j}-\hat{x}_{i}\right) \\
& \quad=W_{j, \rho-1}^{\top}\left(\sum_{r=0}^{\rho} W_{i, r} \varepsilon_{i, r}-W_{j, \rho-1} \varepsilon_{j, \rho-1}\right) .
\end{aligned}
$$

PROOF. First, let us rewrite expression (12) in terms of the estimation error defined in (10):

$$
\begin{aligned}
W_{j, \rho-1}^{\top}\left(\hat{x}_{j}-\hat{x}_{i}\right) & =W_{j, \rho-1}^{\top}\left(\hat{x}_{j}-x+x-\hat{x}_{i}\right) \\
& =W_{j, \rho-1}^{\top}\left(e_{i}-e_{j}\right) .
\end{aligned}
$$

Now, consider the transformed estimation error defined in (11) and let us write the estimation error of agents $i$ and $j$ in the $\varepsilon_{i, \rho}, \varepsilon_{j, \rho}$ coordinates, which yields that:

$W_{j, \rho-1}^{\top}\left(e_{i}-e_{j}\right)=W_{j, \rho-1}^{\top}\left(\sum_{r=0}^{\ell_{i}+1} W_{i, r} \varepsilon_{i, r}-\sum_{r=0}^{\ell_{j}+1} W_{j, r} \varepsilon_{j, r}\right)$.

According to Lemma 3 (i), we have $W_{j, \rho-1}^{\top} W_{j, r}=$ $0, \forall r \geq 0, r \neq \rho-1$, and from Lemma 3 (ii) we know that $\operatorname{Im}\left(W_{j, \rho-1}\right) \subseteq \operatorname{Im}\left(V_{i, \rho}\right)$ which clearly implies $W_{j, \rho-1}^{\top} W_{i, r}=0, \forall r>\rho$, establishing the result.

The following property introduces the method to design the distributed observer gains. After that, it will be shown that this design guarantees exponential convergence of the estimation errors, as well as it is feasible as long as Assumption 6 is satisfied.

Property 11 (Design of the distributed observer) For every agent $i$, the local observation gain $L_{i}$ and the consensus gains $N_{i, j, \rho}$ are designed in such a way that for all $\rho \in\left\{1, \ldots, \ell_{i}\right\}$ matrices:

$$
\begin{aligned}
& D_{i,(0,0)}=W_{i, 0}^{\top} A W_{i, 0}-L_{i} C_{i} W_{i, 0}, \\
& D_{i,(\rho, \rho)}=W_{i, \rho}^{\top} A W_{i, \rho}-\sum_{j \in \mathcal{N}_{i}} N_{i, j, \rho} W_{j, \rho-1}^{\top} W_{i, \rho},
\end{aligned}
$$

have spectral radius smaller than $\alpha$.

Based on this property, we can now state the main result of this paper.

Theorem 12 Consider plant (1) observed by a set of agents that can measure the local outputs (2), and that 
implement the observer structure defined in (8). If the observer gains satisfy Property 11, then the estimates of all the agents tend exponentially to the actual plant state with convergence rate $\alpha$.

PROOF. Let us write the transformed estimation error dynamics for agent $i$ at hop 0 :

$\varepsilon_{i, 0}^{+}=W_{i, 0}^{\top} x^{+}-W_{i, 0}^{\top} \hat{x}_{i}^{+}=\left[W_{i, 0}^{\top} A W_{i, 0}-L_{i} C_{i} W_{i, 0}\right] \varepsilon_{i, 0}$.

Thus, since gain $L_{i}$ is designed in such a way that matrix (13) has spectral radius smaller than $\alpha$, then the estimation error of the locally observable modes of agent $i$ tends exponentially to zero with the decay rate $\alpha$. Note that the locally observable states of each agent are completely decoupled from the unobservable ones.

The second part of the proof consists in proving that, if (14) have spectral radius smaller than $\alpha$, then also the estimation error of the rest of the modes converges to zero exponentially fast with rate $\alpha$. Let us write the transformed estimation error dynamics for an agent $i$ at hop $\rho$, with $\rho \geq 1$, using the orthogonality in Lemma 3 (i):

$$
\begin{aligned}
\varepsilon_{i, \rho}^{+} & =W_{i, \rho}^{\top}\left(A e_{i}-W_{i, \rho} \sum_{j \in \mathcal{N}_{i}} N_{i, j, \rho} W_{j, \rho-1}^{\top}\left(\hat{x}_{j}-\hat{x}_{i}\right)\right) \\
& =W_{i, \rho}^{\top} A \sum_{r=0}^{\rho} W_{i, r} \varepsilon_{i, r}-\sum_{j \in \mathcal{N}_{i}} N_{i, j, \rho} W_{j, \rho-1}^{\top}\left(\hat{x}_{j}-\hat{x}_{i}\right),
\end{aligned}
$$

where $W_{i, \rho}^{\top} W_{i, \rho}=I$ and Lemma 8 has been used to obtain $W_{i, \rho}^{\top} A \sum_{r=\rho+1}^{\ell_{i}+1} W_{i, r}=0$. Next, thanks to Lemma 10, after some manipulations it is possible to rewrite the equation above as:

$$
\varepsilon_{i, \rho}^{+}=\sum_{r=0}^{\rho} D_{i,(\rho, r)} \varepsilon_{i, r}+\sum_{j \in \mathcal{N}_{i}} N_{i, j, \rho} \varepsilon_{j, \rho-1}
$$

with

$$
D_{i,(\rho, r)}:=\left(W_{i, \rho}^{\top} A-\sum_{j \in \mathcal{N}_{j}} N_{i, j, \rho} W_{j, \rho-1}^{\top}\right) W_{i, r},
$$

which extends and completes the equations (13) and (14). From (15), it can be seen that the evolution of the estimation error of agent $i$ at hop $\rho$ depends on the estimation error of that agent at the previous hops and the estimation error of the neighbors at hop $\rho-1$, thus revealing a cascade structure.

We are now in the position to create a vector that stacks the estimation errors of all the agents involved in the network at each hop $\rho$ :

$$
\varepsilon_{\rho}:=\operatorname{col}\left(\varepsilon_{i, \rho}\right)_{i \in \mathcal{V}: \ell_{i}+1 \geq \rho}, \quad \forall \rho \in\{0, \ldots, \bar{\ell}\}
$$

where $\bar{\ell}=1+\max _{i \in \mathcal{V}} \ell_{i}$.

Combining (15) and (17), we generate an expression of the estimation error evolution of all the agents of the network at every hop $\rho$. This leads to:

$$
\left[\begin{array}{c}
\varepsilon_{\bar{\ell}} \\
\vdots \\
\varepsilon_{1} \\
\varepsilon_{0}
\end{array}\right]=\left[\begin{array}{cccc}
\triangle_{\bar{\ell}} & \cdots & \star & \star \\
\vdots & \ddots & \vdots & \vdots \\
0 & \cdots & \triangle_{1} & \star \\
0 & \cdots & 0 & \triangle_{0}
\end{array}\right]\left[\begin{array}{c}
\varepsilon_{\bar{\ell}} \\
\vdots \\
\varepsilon_{1} \\
\varepsilon_{0}
\end{array}\right]
$$

where $\star$ represents some possibly nonzero terms given by (15). It is worth pointing out that according to (15) the diagonal terms of (18) for $\rho \geq 0$ are block diagonal terms with the next structure:

$$
\triangle_{\rho}=\left[\begin{array}{ccc}
D_{1,(\rho, \rho)} & \cdots & 0 \\
\vdots & \ddots & \vdots \\
0 & \cdots & D_{p,(\rho, \rho)}
\end{array}\right]
$$

where $\{1, \ldots, p\} \in \mathcal{V}$, and $D_{i,(\rho, \rho)}$ for $\rho=\left\{1, \ldots, \ell_{i}\right\}$ are defined in (13)-(14) (see also (16)), that is, $D_{i,\left(\ell_{i}+1, \ell_{i}+1\right)}=\bar{V}_{i, \ell_{i}}^{\top} A \bar{V}_{i, \ell_{i}}$ and $D_{i,(\rho, \rho)}$ is the empty matrix for all $\rho \in\left\{\ell_{i}+2, \ldots, \bar{\ell}\right\}$.

Thus, the eigenvalues of the upper triangular matrix in (18) are the eigenvalues of the corresponding matrices placed in its diagonal, which are defined in (19). Finally, it is clear that, if matrices (13) and (14) have spectral radius smaller than $\alpha$ for every agent and hop considered, then the matrix exposed in (18) also has a spectral radius smaller than $\alpha$, and consequently the estimation error of every agent tends exponentially to zero with speed of convergence $\alpha$.

Remark 13 The distributed observer design in this paper has the advantage of inducing linear error dynamics for which we may obtain quadratic Lyapunov certificates. Then we may consider robust extensions of the nominal exponential stability established in Theorem 12 by relying on the intrinsic robustness of Lyapunov-based results. Among other things, this may comprise taking into account sufficiently rare packet losses and their Lyapunov characterization as in [9], which would clearly not destroy the established exponential convergence. Additionally, we may consider partially desynchronized nodes or sufficiently small delays in a sampled-data context where plant (1) is the sampled version of a continuous-time dynamics. Such extensions are left as future work. 
Other non-immediate extensions concern the resilience of the observer to time-varying graphs [25] or switching networks [23], and cyber-attacks on sensors [16].

It is important to note that, according to the transformation made to the system, the proposed structure for the estimators decomposes the influence of the observation gains, $L_{i}$, which only affects the locally observable subspace, from the influence of the consensus gains, $N_{i, j, \rho}$, which has an effect on the locally unobservable subspace.

It is well-known that there always exists a gain $L_{i}$ able to stabilize pair $\left(C_{i} W_{i, 0}, W_{i, 0}^{\top} A W_{i, 0}\right)$ with arbitrary spectral radius. However, it is necessary to prove the existence of matrices $N_{i, j, \rho}$ that induce the same properties on matrices (14). This is proved below.

Theorem 14 (Design feasibility) It is always possible, under Assumption 6, to find a set of matrices $L_{i}$ and $N_{i, j, \rho}$ that satisfy Property 11.

PROOF. The existence of $L_{i}$ is a well-known consequence of observability of pair $\left(C_{i} W_{i, 0}, W_{i, 0}^{\top} A W_{i, 0}\right)$ (see, e.g., the dual statement in $[8$, Th 12.7$])$. In what regards (14), let us fix an arbitrary $i$ and $\rho \leq \ell_{i}$ and rewrite the matrix in (14) in the following compact form:

$$
W_{i, \rho}^{\top} A W_{i, \rho}-\bar{N}_{i, \rho} \Lambda_{i, \rho}
$$

where

$\bar{N}_{i, \rho}=\operatorname{col}\left(N_{i, j, \rho}^{\top}\right)_{j \in \mathcal{N}_{i}}^{\top}$,

$\Lambda_{i, \rho}=\operatorname{col}\left(W_{j, \rho-1}^{\top}\right)_{j \in \mathcal{N}_{i}} W_{i, \rho}$.

To complete the proof it is enough to show that pair $\left(\Lambda_{i, \rho}, W_{i, \rho}^{\top} A W_{i, \rho}\right)$ is observable and apply the same reasoning used for (13).

From the Popov-Belevitch-Hautus test (see, e.g., [8, Th 15.9]) pair $\left(\Lambda_{i, \rho}, A_{i, \rho}^{o}\right):=\left(\Lambda_{i, \rho}, W_{i, \rho}^{\top} A W_{i, \rho}\right)$ is observable if and only if:

$$
\operatorname{rank}\left[\begin{array}{c}
A_{i, \rho}^{o}-\lambda I \\
\Lambda_{i, \rho}
\end{array}\right]=n_{i, \rho}, \quad \forall \lambda \in \sigma\left(A_{i, \rho}^{o}\right) .
$$

This condition can be guaranteed if:

$$
\operatorname{rank}\left[\Lambda_{i, \rho}\right]=n_{i, \rho},
$$

which clearly implies (21). From Lemma 3, we know that:

$$
\operatorname{Im}\left(W_{i, \rho}\right) \subseteq \bigoplus_{j \in \mathcal{N}_{i}} \operatorname{Im}\left(W_{j, \rho-1}\right)
$$

which implies that $\operatorname{rank}\left(W_{i, \rho}\right) \leq \operatorname{rank}\left(\operatorname{col}\left(W_{j, \rho-1}^{\top}\right)_{j \in \mathcal{N}_{i}}\right)$ Finally, using the fact that $W_{i, \rho}$ is a full rank matrix with $\operatorname{rank}\left(W_{i, \rho}\right)=n_{i, \rho}$ it is a simple matter to check that $\operatorname{rank}\left(\operatorname{col}\left(W_{j, \rho-1}^{\top}\right)_{j \in \mathcal{N}_{i}} W_{i, \rho}\right)=\operatorname{rank}\left(\Lambda_{i, \rho}\right)=$ $\operatorname{rank}\left(W_{i, \rho}\right)=n_{i, p}$, which establishes (22) and completes the proof.

\section{Continuous-time construction}

The design technique proposed in this paper can be easily extended to the continuous-time case. This section summarizes its adaptation to this case.

The state-space representation for the continuous-time linear time invariant system is defined as follows:

$$
\begin{aligned}
& \dot{x}=A x, \\
& y_{i}=C_{i} x \quad \forall i \in \mathcal{V} .
\end{aligned}
$$

The subspaces definition as well as Assumption 6 in Section 2 are valid also for the continuous-time case. Regarding the observer structure described in Section 3, the continuous-time expression is given by:

$$
\begin{aligned}
\dot{\hat{x}}_{i} & =A \widehat{x}_{i}+W_{i, 0} L_{i}\left(y_{i}-\widehat{y}_{i}\right)+ \\
& +\sum_{\rho=1}^{\ell_{i}} \sum_{j \in \mathcal{N}_{i}} W_{i, \rho} N_{i, j, \rho} W_{j, \rho-1}^{\top}\left(\widehat{x}_{j}-\widehat{x}_{i}\right) .
\end{aligned}
$$

The lemmas and propositions introduced in Section 4 also apply to this case. Nevertheless, Property 11 must be redefined to design local and consensus gains ensuring convergence abscissa equal to $\alpha$ for the matrices (13) and (14) with $\alpha \in(-\infty, 0)$. Finally, Theorems 12 and 14 apply in both cases.

\section{Distributed design and operation}

This section presents an algorithm to build the estimation structure of each agent $i$. This structure depends on integer and matrices $W_{i, \rho}, \rho=1, \ldots, \ell_{i}$, which should be determined before designing the observer gains $\left(L_{i}, N_{i, j, \rho}\right)$ according to the previous section. Finally, in the running phase, the agents estimate online (distributedly) the plant state $x$. These three phases are clarified below.

Distributed observer setup. In this phase, we design $\ell_{i}$ and matrices $W_{i, \rho}$. First, each agent identifies its locally observable subspace through its output matrix and the plant dynamics, and constructs matrices $V_{i, 0}=W_{i, 0}$. Secondly, each agent exchange its $W_{i, 0}$ matrix with its neighbors. These matrices are used to define the 1-hop observable subspace and to construct matrices $W_{i, 1}$. The algorithm is repeated until hop $\rho=\ell_{i}$ is reached. Note that whenever $\ell_{i}$ is not known, it can be assessed locally 
by computing an observable decomposition and checking whether the $\rho$-hop unobservable modes have speed of convergence faster than $\alpha$. The pseudocode of the design algorithm is given next:

- For every agent $i$ do:

a. Compute $\mathcal{O}_{i, 0}$ and construct matrix $W_{i, 0}$. Set $\rho=0$.

b. Perform the two steps:

- Exchange $W_{i, \rho}$ with the neighbors.

- Construct $\mathcal{O}_{i, \rho+1}$ and construct matrix $W_{i, \rho+1}$.

c. If the $\rho$-hop unobservable modes have speed $\alpha$, then stop and set $\ell_{i}=\rho$. Otherwise increment $\rho$ and go to $(b)$.

d. Exchange $\ell_{i}$ with the neighborhood $\mathcal{N}_{i}$.

Summarizing, in the firs phase each agent $i$ exchanges with its neighbors matrices $W_{i, \rho}$ for $\rho=\left\{0, \ldots, \ell_{i}\right\}$. Due to the fact that, from (7), the set of all these matrices, together with $\bar{V}_{i, \ell_{i}}$ forms the transformation matrix $T_{i}$, it is clear that the exchange of information, in terms of transmitted scalars, is at most $n^{2}$.

Gain selection phase. In this phase each agent selects gains $\left(L_{i}, N_{i, j, \rho}\right)$ in such a way that Property 11 holds. This selection does not require any information exchange because for each agent $i$, matrices $W_{i, \rho}$ and matrices $W_{j, \rho}$ of all the neighbors $j \in \mathcal{N}_{i}$ have been selected and stored in the previous phase.

Running phase. This is the online phase where the distributed observer estimates state $x$. Here, according to the observer structure (8), each agent $i$ will exchange with each neighbor $j \in \mathcal{N}_{i}$ a portion of the state defined by $W_{i, \rho-1}^{\top} \hat{x}_{i}$ for every $\rho \in\left\{1, \ldots, \ell_{j}\right\}$, whose size, according to $(7)$, is at most equal to $n$.

\section{Simulations}

In this section a simulation example is presented in order to show the effectiveness of the proposed observer.

Example 1. The algorithm presented will be compared with the observer structure introduced in [11]. Consider the following continuous-time system:

$$
\dot{x}=\left[\begin{array}{cccc}
0 & 1 & 0 & 0 \\
-1 & 0 & 0 & 0 \\
0 & 0 & 0 & 2 \\
0 & 0 & -2 & 0
\end{array}\right] x
$$

which is being observed by four agents in such a way that $y_{1}=x_{1}, y_{2}=x_{2}, y_{3}=x_{3}$ and $y_{4}=x_{4}$. A cyclic topology for the connection of the agents is considered, namely the graph is composed by edges $(1,2),(2,3)$,
$(3,4),(4,1)$.To carry out the observer design, the system has been discretized with a sampling time of $1 \mathrm{~s}$.

Figure 2 shows the evolution of the plant state modes and the agent 1 estimates for the proposed distributed observer. Note that for this agent, a local design is made to estimate $x_{1}$ while the rest of the states are estimated thought the information provided by its neighborhood. The design has been carried out placing the local and consensus poles around -3 .

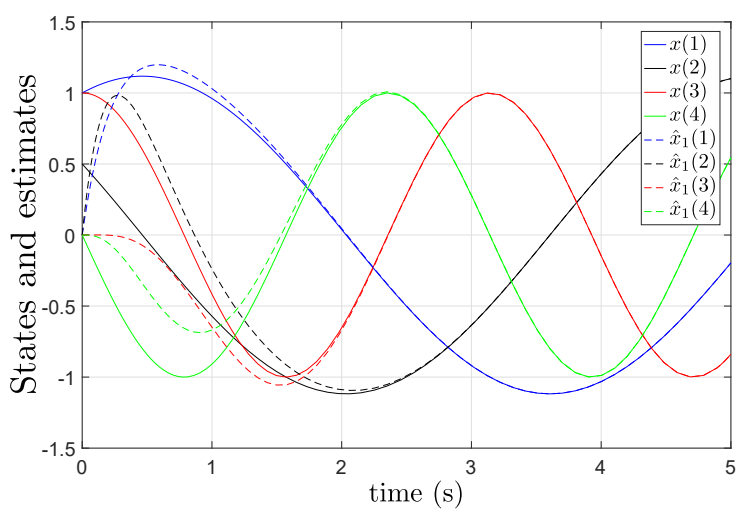

Fig. 2. State of the plant in solid lines and estimates of agent 1 in dashed lines.

To provide a comparison between the estimation performance of the proposed observer and that corresponding to [11], the simulation parameters are set as in the simulation examples provided in that work. Figure 3 shows the evolution of the total error for both structures, which is defined as the average of the 2-norm of the estimation error of each agent:

$$
\bar{e}(t)=\frac{1}{4} \sum_{i=1}^{4}\left\|x(t)-\hat{x}_{i}(t)\right\|_{2} .
$$

Different simulations have been provided regarding the convergence rate fixed by $\alpha$. The observer parameters for the other algorithm has been selected from the simulation example in [11]. Finally, the initial value for the estimations of every state and agent is zero.

It is worth pointing out that the design method proposed in Property 11 makes it easy to place conveniently the estimation error dynamics eigenvalues, taking into account the observable modes at its corresponding hops, in such a way that the convergence can be accelerated, as shown in Figure 3.

\section{Conclusions}

The observer structure presented in this paper introduces a novel method to design and analyze the distributed estimation problem. By decomposing the state- 


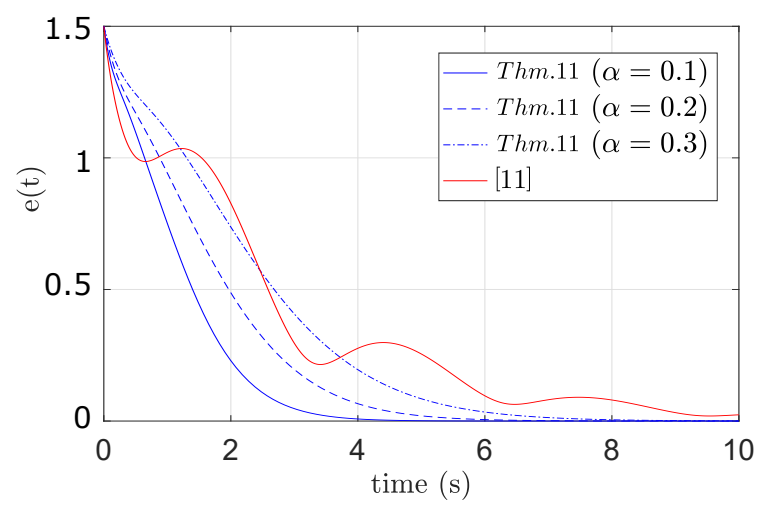

Fig. 3. Evolution of the total error defined in (23) for the algorithm presented in this paper and the observer presented in [11].

space of each agent in locally observable and unobservable subspaces, the last one composed by the innovation introduced by each neighbor at each hop, a distributed design method for the observers has been developed. This design can be carried out through using simple pole placement algorithms, and allows one to adjust the convergence rate. Stability of the presented observer structure has been proven, together with a feasibility condition requiring only necessary conditions for distributed detectability.

Future work will include the design and performance analysis of this observer when communication delays or packet losses are taken into account, as well as the study of optimal and robust designs for noisy measurements and disturbance rejection.

\section{References}

[1] G. Battistelli and L. Chisci. Stability of consensus extended Kalman filter for distributed state estimation. Automatica, 68:169 - 178, 2016.

[2] R. Carli, A. Chiuso, L. Schenato, and S. Zampieri. Distributed Kalman filtering based on consensus strategies. IEEE Journal on Selected Areas in Communications, 26(4):622-633, 2008.

[3] F. S. Cattivelli and A. H. Sayed. Diffusion strategies for distributed Kalman filtering and smoothing. IEEE Transactions on Automatic Control, 55(9):2069-2084, 2010.

[4] A.R. del Nozal, L. Orihuela, and P. Millán. Distributed consensus-based Kalman filtering considering subspace decomposition. In 20th IFAC World Congress, volume 50, pages $2494-2499$, Toulouse, July 2017.

[5] M. Doostmohammadian and U. A. Khan. On the genericity properties in distributed estimation: Topology design and sensor placement. IEEE Journal of Selected Topics in Signal Processing, 7(2):195-204, 2013.

[6] M. Doostmohammadian and U. A. Khan. Graph-theoretic distributed inference in social networks. IEEE Journal of Selected Topics in Signal Processing, 8(4):613-623, 2014.

[7] W. Han, H. L. Trentelman, Z. Wang, and Y. Shen. A simple approach to distributed observer design for linear systems. IEEE Transactions on Automatic Control. Early access 2018.
[8] J. P. Hespanha. Linear systems theory. Princeton university press, 2009.

[9] J. P. Hespanha, D. Liberzon, and A. R. Teel. Lyapunov conditions for input-to-state stability of impulsive systems. Automatica, 44(11):2735-2744, 2008.

[10] H. Ji, L. Lewis, Z. Hou, and D. Mikulski. Distributed information-weighted Kalman consensus filter for sensor networks. Automatica, 77:18 - 30, 2017.

[11] T. Kim, H. Shim, and D. D. Cho. Distributed Luenberger observer design. In Proceedings of the IEEE Conference on Decision and Control (CDC), pages 6928-6933, Las Vegas, December 2016.

[12] P. Millán, A. R. del Nozal, L. Zaccarian, L. Orihuela, and A. Seuret. Distributed implementation and design for state estimation. In 20th IFAC World Congress, volume 50, pages 6483 - 6488, Toulouse, July 2017.

[13] P. Millán, L. Orihuela, C. Vivas, and F. R. Rubio. Distributed consensus-based estimation considering network induced delays and dropouts. Automatica, 48(10):2726-2729, 2012.

[14] P. Millán, L. Orihuela, C. Vivas, F. R. Rubio, D. V. Dimarogonas, and K. H. Johansson. Sensor-networkbased robust distributed control and estimation. Control Engineering Practice, 21(9):1238-1249, 2013.

[15] A. Mitra and S. Sundaram. Distributed observers for LTI systems. IEEE Transactions on Automatic Control. Early access 2018.

[16] A. Mitra and S. Sundaram. Secure distributed state estimation of an LTI system over time-varying networks and analog erasure channels. In Proceedings of the IEEE American Control Conference (ACC), pages 6578 - 6583, Milwaukee, 2018.

[17] R. Olfati-Saber. Distributed Kalman filtering for sensor networks. In Proceedings of the IEEE Conference on Decision and Control (CDC), pages 5492-5498, LA, December 2007.

[18] A. N. Michel P. J. Antsaklis. A linear systems primer. Springer, 2007.

[19] S. Park and N. C. Martins. Necessary and sufficient conditions for the stabilizability of a class of LTI distributed observers. In Proceedings of the IEEE Conference on Decision and Control (CDC), pages 7431-7436, Hawaii, December 2012.

[20] S. Park and N. C. Martins. Design of distributed LTI observers for state omniscience. IEEE Transactions on Automatic Control, 62(2):561-576, Feb 2017.

[21] A. H. Sayed. Adaptive networks. Proceedings of the IEEE, 102(4):460-497, 2014.

[22] J. Thia, Y. Yuan, L. Shi, and J. Goncalves. Distributed Kalman Filter with minimum-time covariance computation. In Proceedings of the IEEE Conference on Decision and Control (CDC), pages 1995-2000, Florence, December 2013.

[23] V. Ugrinovskii. Distributed robust estimation over randomly switching networks using $H_{\infty}$ consensus. Automatica, 49(1):160 - 168, 2013.

[24] L. Wang and A. S. Morse. A distributed observer for a timeinvariant linear system. In Proceedings of the IEEE American Control Conference (ACC), pages 2020-2025, Seattle, May 2017.

[25] L. Wang, A. S. Morse, D. Fullmer, and J. Liu. A hybrid observer for a distributed linear system with a changing neighbor graph. In Proceedings of the IEEE Conference on Decision and Control (CDC), pages 1024-1029, Melbourne, December 2017. 